\title{
Avaliação de Desempenho e Market Timing: O Índice de Habilidade
}

\author{
Ney Roberto Ottoni de Brito*
}

\section{Resumo}

MERTON [1981] formaliza a análise da criação de valor por gestores no contexto geral de seleção entre ações e títulos de renda fixa através de market timing. Partindo dos fundamentos conceituais por ele desenvolvidos, HENRIKSON e MERTON [1981] prosseguem para definir testes gerais de avaliação de desempenho de gestores e fundos no contexto de seleção por market timing. BRITO, BONA E TACIRO [2003] generalizam as análises de MERTON [1981] e HENRIKSON \& MERTON [1981] para o caso de fundos de gestão ativa com uma carteira benchmark passiva claramente definida. Neste contexto generalizado de gestão ativa, um novo índice específico - o Índice de Habilidade de Brito (IHB) - é proposto para a avaliação de desempenho e de eficiência em market timing de fundos de gestão ativa. Este trabalho prossegue, então, para avaliar o desempenho e a eficiência de market timing do segmento de fundos derivativos no Brasil no passado recente utilizando o IHB. Uma amostra de 32 fundos representativos do segmento de derivativos com uma janela de 90 dias úteis com cotações disponíveis em 31/10/1999 foi levantada para testes empíricos de avaliação de desempenho pelo IHB, adotando-se aplicações no CDI como carteira benchmark passiva. Os resultados obtidos indicam a significância ao nível de 5\% dos IHB's de dez fundos da amostra. Destes fundos, sete apresentam também significância ao nível de 1\%. Em síntese, os resultados indicam uma predominância de gestores com capacidade de previsão e IHB's não significantes no segmento de fundos derivativos no Brasil, no período examinado. Poucos gestores apresentam evidência empírica de capacidade de previsão significante.

Palavras chave: Investimentos, Gestão de Investimentos, Avaliação de Desempenho, Market Timing .

Código JEL: G10, G24.

\footnotetext{
${ }^{*}$ Sócio-Diretor da Ney O. Brito \& Associados, Rio de Janeiro, Brasil e foi Professor Titular de Finanças da Universidade Federal do Rio de Janeiro. O autor agradece o apoio geral da Área de Mercado de Capitais do Banco Itaú.
}

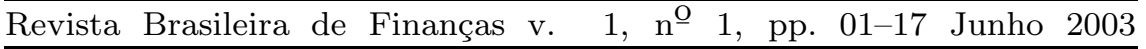




\begin{abstract}
MERTON [1981] examines the creation of value by fund managers selecting between stocks and fixed income instruments through market timing. HENRIKSON and MERTON [1981] proceed to propose empirical tests of funds and manager performance in market timing. BRITO, BONA and TACIRO [2003] generalize the results of MERTON [1981] and HENRIKSON and MERTON [1981] for actively managed funds with a clearly defined benchmark portfolio. In the generalized context of active portfolio management, this paper proposes a new index - the Skill Index of Brito (SIB) - to measure the performance and efficiency in market timing of actively managed funds. The paper proceeds to test the performance and skill of hedge funds in Brazil using the SIB. A representative sample of 32 hedge funds with a window of 90 trading days on October 31, 1999 was obtained. The empirical tests of performance and skill use the interbank borrowing and lending rate as the passive benchmark. The results indicate the significance at the $5 \%$ level of the SIB for ten hedge funds in the sample. Among them seven funds also have shown significance at the $1 \%$ level. In sum the, results indicate a majority of hedge funds with no significant skill in the Brazilian market in the examined period.
\end{abstract}

\title{
1. Introdução.
}

A avaliação de desempenho de fundos e carteiras de investimentos no contexto de eficiência de market timing foi inicialmente proposta por TREYNOR \& MAZUY [1966] através da inclusão de um termo quadrático na regressão convencional de JENSEN [1968] para avaliação de desempenho. Mais precisamente, os autores propõem que a eficiência em market timing seja avaliada pela significância do coeficiente $c$ na regressão:

$$
E X_{X}=a+b E X_{M}+c E X_{M}^{2}+\epsilon
$$

onde

$E X_{X}=r_{X}-r_{F}=$ retorno excessivo do fundo ou carteira $X$ sobre o título de renda fixa sem risco;

$E X_{M}=r_{M}-r_{F}=$ retorno excessivo da carteira de mercado sobre o título de renda fixa sem risco. 
O termo quadrático iria captar a habilidade do gestor do fundo ou da carteira em administrar o market timing em relação ao mercado. Se o gestor espera que renda fixa tenha um desempenho superior ao mercado, ele iria deslocar-se para renda fixa obtendo um retorno diferencial em relação ao mercado, que estaria associado a um termo quadrático positivo. No caso inverso, se espera que o mercado venha a ter um desempenho superior ao título de renda fixa sem risco, então o gestor deslocaria sua carteira para o mercado de ações, obtendo um retorno diferencial em relação ao título de renda fixa que também se expressaria por um termo quadrático positivo. Nos dois casos, existiria um termo quadrático positivo e o coeficiente $c$ seria significante se a capacidade de market timing do gestor fosse superior.

Mais recentemente, MERTON [1981] formalizou a análise da criação de valor por gestores no contexto geral de seleção entre ações e títulos de renda fixa por market timing. Esta formalização parte do Modelo Binomial em dois estágios em que o primeiro estágio está associado às probabilidades de ocorrência de mercados de "alta" ou de "baixa" - rentabilidade de ações superior ou inferior à renda fixa, respectivamente. O segundo estágio está associado à probabilidade condicional de acerto de previsão do gestor. Nesta modelagem, MERTON [1981] desenvolve uma definição formal da habilidade de gestores, associada à sua capacidade de previsão correta em mercados de "alta" e em mercados de "baixa". Mais ainda, limites para a medida de habilidade são definidos sob a condição de que o gestor venha a criar valor para os investidores de sua carteira ou fundo.

Partindo dos fundamentos concentuais desenvolvidos por MERTON[1981], HENRIKSON e MERTON[1981] prosseguem para formalizar testes gerais de avaliação de desempenho dos gestores e fundos no contexto de seleção por market timing. Partindo do modelo binomial geral em dois estágios, estes testes 
são formalizados através da distribuição hipergeométrica. Entretanto, no caso especial em que os gestores têm igual probabilidade de acerto em mercados de "alta" e de "baixa", estes testes reduzem-se à distribuição geométrica associada ao modelo binomial simples.

BRITO, BONA E TACIRO [2003] generalizam as análises de MERTON [1981] e HENRIKSON \& MERTON [1981] para o caso de fundos de gestão ativa com uma carteira benchmark passiva claramente definida. Neste contexto, a habilidade em market timing do gestor envolve a seleção de uma posição com apostas ativas ou uma posição na carteira benchmark passiva. Os autores prosseguem ainda para mostrar que o segmento de fundos derivativos no Brasil parece ajustar-se a uma forma especial do Modelo Binomial Simples - o Modelo Binomial Reduzido. Neste modelo, os ganhos / perdas associados aos acertos / erros do gestor possuem distribuições simétricas, ou seja, distribucionalmente os ganhos e perdas parecem diferir apenas no sinal. No contexto do Modelo Binomial Reduzido, os autores prosseguem para obter estimativas de risco e retorno de fundos de gestão ativa, em geral, e de fundos de derivativos no mercado brasileiro, em particular.

No contexto de gestão ativa generalizado por BRITO, BONA \& TACIRO [2003], testes de avaliação de desempenho e de eficiência em market timing podem ser desenvolvidos para fundos de gestão ativa. Este trabalho prossegue, então, para avaliar o desempenho e a eficiência de market timing do segmento de fundos derivativos no Brasil no passado recente, utilizando o contexto do Modelo Binomial simples e a distribuição geométrica. Um índice específico - o Índice de Habilidade de Brito (IHB) - é proposto para a avaliação de desempenho e de eficiência em market timing de fundos de gestão ativa.

O capítulo II examina os fundamentos conceituais da modelagem de gestores no contexto de market timing, definindo sua 
habilidade através da capacidade de previsão. O capítulo III formaliza os testes de significância de habilidade e capacidade de previsão que representam também um teste de avaliação de desempenho dos gestores dos fundos. O capítulo IV apresenta os resultados dos testes para o segmento de fundos derivativos no Brasil no passado recente. Finalmente, o capítulo V sintetiza as principais conclusões deste trabalho.

\section{Fundamentos Conceituais da Modelagem de Gesto- res.}

MERTON [1981] desenvolveu um modelo básico conceitual para análise de market timing, onde o gestor do fundo, com base em sua capacidade de previsão, aloca recursos entre ações e títulos de renda fixa, de acordo com a sua previsão de superior desempenho. A habilidade do gestor é definida no contexto de probabilidades condicionais no modelo binomial em dois estágios, como apresentado na Figura 1.

No primeiro estágio, os investidores observam as probabilidades de que os títulos de renda fixa $\left(R_{F}\right)$ irão ter um desempenho superior ao das ações $(A)$ ou vice-versa. No segundo estágio, os investidores examinam a probabilidade de que o gestor irá prever corretamente se títulos de renda fixa ou ações irão oferecer um desempenho superior. Mais precisamente, representando-se as previsões do gestor em $(t-1)$ pela variável binominal $\delta(t)$ onde:

$\delta(t)=1$ quando espera-se que ações venham a oferecer desempenho superior em $t: A_{t}>R_{F t}$;

$\delta(t)=0$ quando espera-se que títulos de renda fixa venham a oferecer um desempenho superior em $t: R_{F t} \geq A_{t}$.

Então, as probabilidades condicionais podem ser definidas como: 
$p_{1}(t)=\operatorname{prob}\left[\delta(t)=1 \mid A_{t}>R_{F t}\right]=$ a probabilidade de que o gestor corretamente prevê que ações iriam ter um desempenho superior aos títulos de renda fixa;

$p_{2}(t)=\operatorname{prob}\left[\delta(t)=0 \mid R_{F t} \geq A_{t}\right]=$ a probabilidade de que o gestor corretamente prevê que títulos de renda fixa terão um desempenho superior às ações.

\section{Figura 1}

Modelo Binomial em Dois Estágios

MERTON [1981] prossegue para mostrar uma condição necessária para que a previsão do gestor tenha um valor positivo para os investidores:

$$
p_{1}(t)+p_{2}(t)>1
$$

Isto permite obter-se uma definição formal de habilidade do gestor como:

Habilidade em Market Timing $=p_{1}(t)+p_{2}(t)$.

Na Figura 1, os resultados excessivos aleatórios são expressos como ganhos de ações em relação à renda fixa, ou vice-versa, 
no caso de previsões corretas. No caso de previsões incorretas, os resultados incorretos representam perdas relativas.

Esta definição geral de habilidade em market timing permite a gestores terem diferentes capacidades de previsão em mercados de "alta" e em mercados de "baixa" ao utilizar probabilidades condicionais. Entretanto, se a capacidade de previsão do gestor é idêntica em mercados de "alta" e em mercados de "baixa", então $p_{1}(t)=p_{2}(t)$, o que implica que probabilidades condicionais não são relevantes. Neste caso, gestores podem ser modelados no contexto do Modelo Binomial Simples ${ }^{1}$ representado na Figura 2, onde:

$p=$ habilidade do gestor e probabilidade incondicional de uma previsão correta;

$\mu_{G}, \sigma_{G}=$ a média e desvio padrão de ganhos quando a previsão do gestor está correta;

$\mu_{E}, \sigma_{E}=$ média e desvio padrão de perdas quando a previsão do gestor está incorreta.

Na Figura 2, os resultados excessivos representam os ganhos / perdas de ações em relação a renda fixa, ou vice-versa, no caso de acertos / erros do gestor.

No contexto do Modelo Binomial Simples da Figura 2, com suas probabilidades incondicionais, a condição de previsão racional e criação de valor - a relação (1) anterior - é reduzida a:

$$
p>0,5
$$

Ou seja, para criar valor, um gestor precisa acertar mais do que errar.

\footnotetext{
${ }^{1}$ O Modelo Binomial Simples, no contexto de seleção entre ações e renda fixa, é discutido por HENRIKSON \& MERTON [1981]. Eles também prosseguem para desenhar um teste empírico adequado a este contexto de seleção. 
BRITO, BONA \& TACIRO [2003] prosseguem para generalizar todas as relações propostas por MERTON [1981] e HENRIKSON \& MERTON [1981] para o caso de gestão de um fundo ou carteira com uma carteira benchmark passiva claramente definida. Neste contexto, as apostas ativas do gestor do fundo devem ser sempre feitas em relação à sua previsão de desempenho relativo em relação à carteira benchmark passiva. Os autores mostram ainda que gestores com maior habilidade em market timing em relação à carteira benchmark passiva produzem fundos com superior retorno esperado e menor risco.

Neste sentido, a probabilidade $p$ de acerto do gestor em suas apostas ativas em relação à carteira benchmark passiva é um índice adequado para a avaliação de desempenho de um fundo de gestão ativa. Esta medida da avaliação de desempenho representa o Índice de Habilidade de Brito (IHB) proposto neste trabalho.

Figura 2

Estrutura Geral do Modelo Binomial Simples

Os resultados da gestão podem ser modelados no contexto binomial com duas alternativas de possíveis resultados: desempenho superior da aposta ativa na carteira do fundo ou da aposta passiva na carteira benchmark. O Índice de Habilidade de Brito (IHB) é, então, definido como a probabilidade de acerto da aposta ativa do gestor, mantendo-se o contexto do 
Modelo Binomial Simples da Figura 2. No contexto da Figura 2 , os retornos excessivos representam os ganhos / perdas relativos do fundo em relação à carteira benchmark, ou vice-versa, no caso de acerto / erro do gestor.

Aceitando-se a premissa básica de validade do Modelo Binomial Simples, isto é, que as probabilidades condicionais de acerto das apostas do gestor em relação à carteira benchmark são idênticas em mercados de "alta" e em mercados de "baixa", pode-se prosseguir, então, para testes de avaliação de desempenho com base na distribuição geométrica associada ao Modelo Binomial Simples. Observe ainda que a condição de previsão racional e de criação de valor do gestor mantém-se como expresso na relação (2). Ou seja, um gestor racional para criar valor para os investidores de seu fundo deve acertar com probabilidade superior a 0,5 na obtenção de um retorno diferencial positivo em relação à carteira benchmark em suas apostas na carteira do fundo e deve apresentar um Índice de Habilidade de Brito (IHB) superior a 0,5.

\section{Formalização de Teste de Significância de Capaci- dade de Previsão e do Índice de Habilidade de Brito.}

No contexto generalizado de gestão ativa, a distribuição binomial permite o teste da capacidade de previsão - forecasting skills - e do Índice de Habilidade de Brito do gestor de um produto. Definindo-se:

$n=\mathrm{n}^{\underline{\mathrm{O}}}$ total de períodos examinados;

$k=\mathrm{n}^{\mathrm{O}}$ de períodos que representaram acertos;

$p=$ probabilidade de acerto e Índice de Habilidade de Brito (IHB);

$P(k \mid N, p)=$ probabilidade de existirem $k$ acertos dado que existiram $N$ períodos de gestão com probabilidade de acerto $p$; $\left(\begin{array}{l}N \\ k\end{array}\right)=$ combinação de $N$ em $k$. 
Então, pode-se obter que:

$$
P(k \mid N, p)=\left(\begin{array}{c}
N \\
k
\end{array}\right) p^{k}(1-p)^{N-k}
$$

O que representa a função de probabilidade da binomial correspondente. A função distribuição seria obtida agregando-se esta função probabilidade, observando que $k$ varia entre 0 e $N$ - ou seja, $K \epsilon[0, N]$. Assim, a probabilidade de que $k \leq j$ e a função distribuição seriam obtidas por:

$$
P(k \leq j \mid N, p)=\sum_{k=\emptyset}^{j}\left(\begin{array}{l}
N \\
k
\end{array}\right) p^{k}(1-p)^{N-k}
$$

No caso específico de um gestor sem capacidade de previsão, a probabilidade de acertar ou errar seriam iguais e ambas seriam iguais a 0,5. Neste contexto, as relações (3) e (4) seriam reduzidas, respectivamente, a:

$$
\begin{gathered}
P(k \mid N ; p=0,5)=\left(\begin{array}{c}
N \\
k
\end{array}\right) 0,5^{N} \\
P(k \leq j \mid N ; p=0,5)=\sum_{k=0}^{j}\left(\begin{array}{l}
N \\
k
\end{array}\right) 0,5^{N}
\end{gathered}
$$

E permitem um teste direto do Índice de Habilidade de Brito $p$ do gestor.

Parece razoável prosseguir-se para especificar um teste unicaudal, adotando-se um limite crítico $k^{*}$ a partir do qual ficaria evidenciada a significância estatística da capacidade de previsão do gestor. Adotando-se um nível de confiança estatística $\alpha-$ poderia ser $95 \%$ ou $99 \%$ - o valor crítico $k^{*}$ poderia ser obtido precisamente pela relação (6). Ele seria a solução de:

$$
P\left(k \leq k^{*} \mid N ; p=0,5\right)=\sum_{k=0}^{k^{*}}\left(\begin{array}{c}
N \\
k
\end{array}\right) 0,5^{N}=1-\alpha
$$


E o teste de significância estatística envolveria a simples comparação entre o número de acertos $n$ e o limite $k^{*}$. Se $n>k^{*}$ existe evidência de um Índice de Habilidade de Brito significante e se $n \leq k^{*}$ a evidência indicará um Índice de Habilidade de Brito não significante do gestor.

A obtenção do limite $k^{*}$ pela relação precisa (7) depende de somatórios e das fórmulas com fatoriais de combinações. Esta complicação pode ser evitada utilizando-se a aproximação normal da binomial. Cabe observar que a binomial representada pelas relações (5) e (6) tem a importante propriedade de ser simétrica. Isto torna a aproximação normal extremamente precisa para amostras acima de 30 observações.

A aproximação da binomial seria feita pela normal com média e desvio padrão definidos por $^{2}$ :

$$
\begin{gathered}
\mu=0,5 N \\
\sigma=\sqrt{0,25 N}
\end{gathered}
$$

Utilizando-se o nível de confiança de $95 \%$ o limite crítico $k^{*}$ para o teste unicaudal seria obtido por:

$$
k_{0,95}^{*}=\mu+1,65 \sigma=0,5 N+1,65 \sqrt{0,25 N}
$$

E seria, então, comparado com o número efetivo de acertos $n$. Para o nível de confiança de $99 \%$, o valor crítico seria obtido por:

$$
k_{0,99}^{*}=0,5 N+2,33 \sqrt{0,25 N}
$$

Os testes do Índice de Habilidade de Brito (IHB) de diversos gestores de produtos derivativos serão conduzidos no capítulo IV, a seguir. Na realidade, como discutido no capítulo II, estes

\footnotetext{
${ }^{2}$ Uma discussão da aproximação normal da binomial pode ser encontrada em BOWKER \& LIEBERMAN [1959]. Eles indicam que a aproximação é particularmente boa para $p$ em torno de 0,5 , como será o caso da presente aplicação. 
são testes da significância do desempenho dos gestores dos fundos.

\section{Resultado dos Testes de Avaliação de Desempenho e de Market Timing no Mercado Brasileiro.}

No contexto do Modelo Binomial Simples, os testes do capítulo anterior podem ser generalizados para examinar avaliação de desempenho e de market timing do gestor de qualquer fundo ou carteira ativo com uma carteira benchmark passiva claramente definida. Definindo-se:

$r_{f t}=$ retorno do fundo no período $t ;$

$r_{b t}=$ retorno na carteira benchmark passiva no período $t$.

Então, a previsão do gestor para o período $t$ pode ser classificada ex-post como correta ou incorreta:

$$
\begin{aligned}
& r_{f t}>r_{b t} \text { implica em uma previsão correta; } \\
& r_{f t} \leq r_{b t} \text { implica em previsão incorreta. }
\end{aligned}
$$

Aplicando-se as condições da relação (3) numa série temporal de retornos da carteira e do benchmark, pode-se prosseguir para estimar todas as variáveis associadas com Modelo Binomial Simples da Figura 2:

$p=$ freqüência de previsões corretas e Índice de Habilidade de Brito do gestor;

$\left\{\left(r_{f t}-r_{f t}\right)^{+}\right\}=$conjunto de observações associadas a ganhos em relação ao benchmark e a previsões corretas;

$\left\{\left(r_{f t}-r_{f t}\right)^{-}\right\}=$conjunto de observações associadas a perdas relativas ao benchmark e a previsões incorretas;

$\mu_{G}, \sigma_{G}=$ a média e desvio padrão de $\left\{\left(r_{f t}-r_{b t}\right)^{+}\right\}$

$\mu_{P}, \sigma_{P}=$ a média e desvio padrão de $\left\{\left(r_{f t}-r_{b t}\right)^{-}\right\}$ 
Com base no Índice de Habilidade de Brito $p$ de previsões corretas e no número de observações associadas a previsões corretas, pode-se prosseguir para testes de significância de desempenho da carteira do fundo e de habilidade de seu gestor em market timing. Os procedimentos dos testes de significância de desempenho e habilidade em market timing foram discutidos e apresentados no capítulo III anterior.

Para se conduzir os testes de significância de desempenho e de habilidade em market timing de gestores, utilizando o Índice de Habilidade de Brito, é conveniente delimitar-se um segmento específico de fundos de gestão ativa como amostra básica. No mercado brasileiro de fundos, o segmento mais crítico em relevância de market timing é o segmento de fundos derivativos. Neste segmento, os gestores não só fazem apostas ativas direcionais mas podem alavancar suas apostas. Neste contexto, delimitou-se como amostra básica para os testes empíricos de market timing os 32 fundos de derivativos disponíveis no mercado em 31 de outubro de 1999 e com uma série completa de observações na janela de 90 dias úteis de cotações e rentabilidade nesta data.

Seguindo uma prática comum de mercado, adotou-se como carteira benchmark passiva para a amostra de fundos derivativos uma aplicação integral na taxa interbancária - o CDI. Neste contexto, o gestor de um fundo derivativo é modelado como optando entre estacionar seus recursos no CDI ou fazer aplicações ativas e direcionais em outros mercados.

Com a amostra de 32 fundos derivativos, suas rentabilidades diárias em uma janela de 90 dias úteis terminando em 31 de outubro de 1999 e com o CDI como benchmark passivo prosseguiu-se para os testes empíricos. Os resultados são apre- 
sentados na Tabela 1, a seguir.

A primeira coluna da Tabela 1 apresenta o número de acertos com ganhos do gestor em relação ao CDI e a segunda coluna apresenta o Índice de Habilidade de Brito (IHB) estimado como a freqüência de acertos em relação as 90 observações disponíveis. A terceira coluna apresenta a probabilidade de que o número de acertos observados possa ser atribuído a eventos aleatórios não associados à superior capacidade de previsão do gestor e a quarta coluna apresenta a significância estatística dos IHB's ao nível de $5 \%$ e $1 \%$.

Os resultados indicam que dez fundos da amostra apresentaram IHB significante ao nível de 5\%. Mais ainda, sete fundos apresentaram IHB significante ao nível de 1\%. Estes resultados sugerem que uma proporção significativa de fundos derivativos da amostra apresentaram significantes IHB's. Os dez fundos com IHB's significantes ao nível de 5\% tiveram como gestores os seguintes bancos: Itaú, Francês e Brasileiro, HSBC, Liberal, Modal e Pactual. Entretanto, a ampla maioria dos fundos do segmento não apresentou a significância de IHB's ${ }^{3}$.

Cabe observar que no período a taxa repo SELIC em geral superou a taxa do CDI. Neste contexto, pode-se argumentar que a taxa SELIC seria um melhor benchmark passivo do que o CDI. A utilização da taxa SELIC como benchmark passivo certamente tenderia a reduzir o número de fundos derivativos com IHB's significantes ${ }^{4}$.

\footnotetext{
${ }^{3} \mathrm{O}$ IHB passou a ser regularmente divulgado ao mercado pelo Sistema Quantum Axis da Quantum-Avaliação de Investimentos. Estimativas atualizadas dos IHB's de fundos podem ser obtidas neste Sistema.

${ }^{4}$ Como o diferencial entre as taxas SELIC e CDI varia ao longo do tempo, seria interessante examinar-se a diferenciação entre os resultados obtidos em diferentes períodos utilizando as duas taxas. Este é um tópico para pesquisas futuras.
} 
Tabela 1

Testes Empíricos de Avaliação de Desempenho e de Capacidade de Previsão

Fundos Derivativos - Janela de 90 dias em 31/10/1999

\begin{tabular}{|c|c|c|c|c|}
\hline FUNDO & $\mathrm{N}^{\mathrm{O}}$ Acertos & IHB's & Distr.Normal & Significância \\
\hline Frances Derivativos & 79 & $87,8 \%$ & $0,00 \%$ & $\mathrm{~S} 1 \%$ \\
\hline Intrepid FIF & 72 & $80,0 \%$ & $0,00 \%$ & $\mathrm{~S} 1 \%$ \\
\hline Itaú Strategy II FIF & 85 & $94,4 \%$ & $0,00 \%$ & $\mathrm{~S} 1 \%$ \\
\hline Societé Derivativos & 85 & $94,4 \%$ & $0,00 \%$ & $\mathrm{~S} 1 \%$ \\
\hline AGF FIF 60 Mix & 37 & $41,1 \%$ & $95,42 \%$ & \\
\hline Atico Hedge 60 & 42 & $46,7 \%$ & $73,65 \%$ & \\
\hline Atico Leverage & 34 & $37,8 \%$ & $98,98 \%$ & \\
\hline Boston Private Port.II & 47 & $52,2 \%$ & $33,66 \%$ & \\
\hline Boston Private Strategy & 52 & $57,8 \%$ & $7,00 \%$ & \\
\hline Garantia High Yield & 44 & $48,9 \%$ & $58,35 \%$ & \\
\hline Garantia Port.Plus & 47 & $52,2 \%$ & $33,66 \%$ & \\
\hline Hedging Griffo Top 60 & 51 & $56,7 \%$ & $10,30 \%$ & \\
\hline Hedging Griffo Verde & 47 & $52,2 \%$ & $33,66 \%$ & \\
\hline HSBC Bam.Priv.Der. & 63 & $70,0 \%$ & $0,01 \%$ & $\mathrm{~S} 1 \%$ \\
\hline Icatu Advanced & 48 & $53,3 \%$ & $26,35 \%$ & \\
\hline IP GAP Hedge & 49 & $54,4 \%$ & $19,95 \%$ & \\
\hline Liberal Dinâmico & 55 & $61,1 \%$ & $1,75 \%$ & $\mathrm{~S} 5 \%$ \\
\hline Liberal High Yield & 54 & $60,0 \%$ & $2,89 \%$ & $\mathrm{~S} 5 \%$ \\
\hline Liberal Moderado 60 & 54 & $60,0 \%$ & $2,89 \%$ & $\mathrm{~S} 5 \%$ \\
\hline Lloyds Mix & 51 & $56,7 \%$ & $10,30 \%$ & \\
\hline Matrix K2 & 44 & $48,9 \%$ & $58,35 \%$ & \\
\hline Matrix Leverage FIF 60 & 40 & $44,4 \%$ & $85,41 \%$ & \\
\hline Modal Advanced & 64 & $71,1 \%$ & $0,00 \%$ & $\mathrm{~S} 1 \%$ \\
\hline Opportunity Deriv.Mod. & 1. 44 & $48,9 \%$ & $58,35 \%$ & \\
\hline Opportunity Plus & 46 & $51,1 \%$ & $41,65 \%$ & \\
\hline Pactual Hedge 60 & 66 & $73,3 \%$ & $0,00 \%$ & $\mathrm{~S} 1 \%$ \\
\hline Patrimonio Dynamic Spe & e.36 & $40,0 \%$ & $97,11 \%$ & \\
\hline Pictet Modal & 44 & $48,9 \%$ & $58,35 \%$ & \\
\hline Plural Extra de Inv.Fin. & . 45 & $50,0 \%$ & $50,00 \%$ & \\
\hline Sul Am.Crescimentum & 45 & $50,0 \%$ & $50,00 \%$ & \\
\hline Sul Am.Proventum & 44 & $48,9 \%$ & $58,35 \%$ & \\
\hline Unibanco Derivativos & 49 & $54,4 \%$ & $19,95 \%$ & \\
\hline
\end{tabular}

Observação: S1\% e S5\% na última coluna indicam significância aos níveis de $1 \%$ e $5 \%$, respectivamente. 


\section{Conclusões.}

Este trabalho formalizou critérios para avaliação de desempenho e da capacidade de previsão - market timing - de fundos de gestão ativa com carteiras benchmark passivas claramente definidas, partindo da generalização dos resultados de MERTON [1981] proposta por BRITO, BONA \& TACIRO [2003].

Utilizando a modelagem dos gestores de fundos ativos como colocando apostas ativas em relação à sua carteira benchmark passiva, este trabalho prossegue para propor uma nova medida de avaliação de desempenho e eficiência de fundos de gestão ativa: o Índice de Habilidade de Brito (IHB). Testes empíricos do Índice de Habilidade de Brito (IHB) são derivados e propostos.

Capacidade de previsão é particularmente crítica no segmento de fundos derivativos. Pela natureza deste produto, seus gestores fazem apostas direcionais com alavancagem. Por tais características, o trabalho prossegue para conduzir os testes do Índice de Habilidade de Brito (IHB) no segmento de fundos derivativos que deveriam apresentar o melhor nível de capacidade de previsão.

Ao se levantar os fundos do segmento com uma janela de 90 dias úteis com cotações disponíveis em 31 de outubro de 1999, obteve-se uma amostra de 32 fundos para a aplicação dos testes empíricos, adotando-se aplicações no CDI como carteira benchmark passiva.

Os resultados obtidos indicaram a significância ao nível de $5 \%$ dos IHB's de dez fundos da amostra. Destes fundos, sete apresentaram também significância ao nível de 1\%. Os fundos derivativos com significantes IHB's eram administrados pelos bancos Itaú, Francês e Brasileiro, HSBC, Modal, Pactual e Liberal. A ampla maioria dos fundos - 22 fundos, mais de 2/3 da amostra - não apresentaram qualquer evidência de IHB's 
superiores.

Em síntese, os resultados indicam uma predominância de gestores com capacidade de previsão e IHB's não significantes no segmento de fundos derivativos no Brasil, no período examinado. Poucos gestores apresentam evidência empírica de capacidade de previsão significante.

Submetido em Abril de 2003. Revisado em Maio de 2003.

\section{References}

Bowker, A. \& Lieberman, G. 1959. Engineering Statistics. Prentice Hall, Englewood Cliffs, New Jersey.

Brito, N., Bona, A. \& Taciro, A. 2003. "Estimating Risk and Return Combinations For New Actively Managed Funds", Manuscrito Não Publicado, Maio.

Henriksson, R. \& Merton, R. 1981. "On Market Timing and Investment Performance. II. Statistical Procedures for Evaluating Forecasting Skills", Journal of Business, 54(4), 515-533.

Merton, R. 1981. "On Market Timing and Investment Performance. I. An Equilibrium Theory of Value for Market Forecasts", Journal of Business, 54(3), 363-406.

Treynor, J. \& Mazuy, M. 1966. "Can Mutual Funds Outguess Market?", Harvard Business Review, 44(4), 131-136. 